\title{
Gating of Sensory Input at Spinal and Cortical Levels during Preparation and Execution of Voluntary Movement
}

\author{
Kazuhiko Seki ${ }^{1,2,3,4}$ and Eberhard E. Fetz ${ }^{4}$ \\ ${ }^{1}$ Department of Neurophysiology, National Institute of Neuroscience, National Center of Neurology and Psychiatry, Tokyo 187-8502, Japan, ${ }^{2}$ Department of \\ Developmental Physiology, National Institute for Physiological Sciences, Aichi 444-8585, Japan, ${ }^{3}$ PRESTO, Japan Science and Technology Agency, \\ Kawaguchi, Saitama 332- 0012, Japan, and ${ }^{4}$ Department of Physiology and Biophysics, and Washington National Primate Research Center, University of \\ Washington, Seattle, Washington 98195-7290
}

All bodily movements stimulate peripheral receptors that activate neurons in the brain and spinal cord through afferent feedback. How these reafferent signals are processed within the CNS during movement is a key question in motor control. We investigated cutaneous sensory-evoked potentials in the spinal cord, primary somatosensory and motor cortex, and premotor cortex in monkeys performing an instructed delay task. Afferent inputs from cutaneous receptors were suppressed at several levels in a task-dependent manner. We found two types of suppression. First, suppression during active limb movement was observed in the spinal cord and all three cortical areas. This suppression was induced by both bottom-up and top-down gating mechanisms. Second, during preparation for upcoming movement, evoked responses were suppressed exclusively in the motor cortical areas and the magnitude of suppression was correlated with the reaction time of the subsequent movement. This suppression could be induced by a top-down gating mechanism to facilitate the preparation and execution of upcoming movement.

\section{Introduction}

Every bodily movement stimulates peripheral sensory receptors that activate neurons in the CNS via sensory pathways. This reafferent feedback is not constant, but is influenced by top-down mechanisms that regulate sensory feedback during movement. Such sensory gating is evident in the reduction of the amplitude of sensory responses during movement and has been proposed as a mechanism to modulate afferent input to the CNS. Sensory gating has been found in multiple regions in the mammalian CNS, including the oculomotor (Thilo et al., 2004), whisker (Urbain and Deschênes, 2007), and orofacial system (Andreatta and Barlow, 2003).

Gating of somatic sensation during voluntary limb movement has been amply documented (Angel and Malenka, 1982; Blakemore et al., 1998; Bays et al., 2006). For example, psychophysical thresholds for detecting tactile stimulation are increased during movement (Milne et al., 1988) and the threshold changes covary with movement parameters (Angel and Malenka, 1982). Somatosensory-evoked potentials in human cerebral cortex are also modulated during movement (Starr and Cohen, 1985). Attenuation of evoked responses is greater during self-initiated than

\footnotetext{
Received Sept. 29, 2011; revised Nov. 23, 2011; accepted Nov. 30, 2011.

Author contributions: K.S. and E.E.F. designed research; K.S. performed research; K.S. analyzed data; K.S. and E.E.F. wrote the paper.

This work was supported by NIH Grants R01 NS 12542 and RR00166, Grant-in-Aid for Scientific Research (KAKENHI), and Japan Science and Technology Agency PRESTO program. We thank Steve Perlmutter for surgical assistance, Tomohiko Takei for attending part of recording, Nobuaki Takahashi and Sara Gilbert for animal care and training, and Larry Shupe and Scott Votaw for computer programming. We also thank Morio Togawa and Masashiro Mori for technical assistance.

The authors declare no competing financial interests.

Correspondence should be addressed to Kazuhiko Seki, Department of Neurophysiology, National Institute of Neuroscience, National Center of Neurology and Psychiatry, 4-1-10gawa-Higashi, Kodaira, Tokyo 187-8502, Japan. E-mail:seki@ncnp.go.jp. DOl:10.1523/JNEUROSCI.4958-11.2012

Copyright $\odot 2012$ the authors $\quad 0270-6474 / 12 / 320890-013 \$ 15.00 / 0$
}

passive movement (Rushton et al., 1981); this attenuation is specific to afferent input from the contralateral limb (Cohen and Starr, 1987). The magnitude of gating is also related to parameters of the motor task (Rushton et al., 1981; Wasaka et al., 2005). Abnormal absence of attenuation has been implicated in clinical syndromes, such as schizophrenia (Shergill et al., 2005) and focal dystonia (Murase et al., 2000).

The sites and roles of somatosensory gating in the preparation and execution of limb movement remain largely unknown. Sensory gating during movements has been found in several levels in the ascending sensory pathway (i.e., dorsal column nuclei, medial lemniscus, thalamus) (Ghez and Pisa, 1972; Coulter, 1974; Tsumoto et al., 1975; Chapman et al., 1988; Hantman and Jessell, 2010). Gating found in the cerebral cortex (Jiang et al., 1990) could be attributed to suppression in these sensory relays, but could also involve corticocortical mechanisms. To resolve the way that the separate sensorimotor regions in the CNS are involved in manifesting and generating sensory gating, it is crucial to evaluate the sensory gating in these different regions under the same experimental conditions.

Our study was designed to elucidate the neural mechanisms that produce sensory gating by probing the evoked responses in cortical and subcortical regions during preparation and execution of active hand movement. We measured the local field potentials evoked by electrical stimulation of a forearm cutaneous nerve and recorded these sensory-evoked potentials (SEPs) in cervical spinal cord (SC), primary sensory cortex (S1), primary motor cortex (M1), and premotor cortex (PM) in monkeys performing a voluntary wrist flexion-extension task with an instructed delay period. We found evidence for two kinds of sensory gating: nonspecific and specific. Nonspecific sensory gat- 
ing was found during active movement and hold, and appeared widely distributed at all recording sites. In contrast, specific sensory gating was found during an instructed delay period exclusively in the motor cortical areas that contribute to preparation and execution of movement.

Preliminary results have been presented in abstract form (Seki and Fetz, 2010).

\section{Materials and Methods}

Subjects. We obtained data from two monkeys (Macaca nemestrina and M. fuscata). Experiments were approved by the Institutional Animal Care and Use Committee at the University of Washington and the Animal Research Committee at the National Institute for Physiological Sciences. During training and recording sessions, the monkeys sat upright in a primate chair with the right arm restrained and elbow bent at $90^{\circ}$. The right hand was held in a cast with the fingers extended and the forearm in the mid-supination/pronation position. The cast holding the monkey's hand was attached to a servomotordriven manipulandum. The left arm was loosely restrained to the chair.

Behavioral paradigm. The monkeys were trained to generate auxotonic (spring-loaded), ramp-and-hold flexion and extension torques about the wrist. Torque controlled the position of a cursor on a video screen in front of the animal (Fig. $1 B$, bottom).

Trials began with the cursor held in a center target window, corresponding to zero torque, for 1.3-1.6 s (rest). Next, the flexion and extension targets were shown to the left and right of the center target. One target was filled transiently ( $0.3 \mathrm{~s}$; cue) indicating the correct movement to be performed at the end of the instructed delay period (delay), signaled by disappearance of the center target (go). No wrist movement occurred during the delay period (between 1.5 and $2 \mathrm{~s}$, randomized) of accepted trials. Following a brief reaction time (RT) after the go signal, the monkey moved (active move) the cursor to the desired target quickly $(<1.5 \mathrm{~s}$ including RT) and held against an elastic load for a period of $1.5 \mathrm{~s}$ (active hold). At the end of the active hold period, the torque target disappeared and the center target reappeared (second go). After a second RT, the monkey relaxed the forearm muscles, allowing the servo-controlled spring to passively return the wrist (Passive Move) to the zero torque position (rest). After keeping the cursor within the center target for $0.8 \mathrm{~s}$, the monkey was rewarded with applesauce (reward) for successful trials.

In this paradigm, the monkeys exerted wrist movements of $31.4 \pm 2.9^{\circ}$ in flexion and $28.6 \pm 3.9^{\circ}$ in extension.

Surgical implants. After training, surgeries were performed aseptically with the animals under $1-1.5 \%$ isoflurane anesthesia. Head stabilization lugs were cemented to the skull with dental acrylic, anchored to the bone via screws. For recording SEPs of spinal cord, a plastic (Ultem 2000; Modern Plastics) recording chamber was implanted over a hemilaminectomy of the lower cervical vertebrae (C4-T1) (Perlmutter et al., 1998). For recording SEPs at cortical sites, a plastic chamber allowing exploration of a $30-\mathrm{mm}-$ diameter cortical area was implanted over the forelimb area on the left hemisphere of each monkey (Fig. $1 A$ ). For recording EMGs, pairs of stainless steel wires (AS632; Cooner Wire) were implanted subcutaneously in 10-12 muscles (Seki et al., 2009). Two nerve cuff electrodes (Haugland, 1996; Seki et al., 2009) were implanted on the superficial radial nerve (SR), midway between elbow and wrist, and separated by $4-5 \mathrm{~cm}$ (Fig. $1 A$ ): a distal bipolar cuff for stimulation and a tripolar cuff for recording orthodromic volleys evoked by stimuli to the distal cuff electrodes.

Data recording. SEPs evoked by electrical stimuli applied to the SR were recorded with glass-insulated tungsten microelectrodes or glass-insulated elgiloy microelectrodes $(0.2-1.0 \mathrm{Mohm})$ while the monkey performed wrist flexion and extension movements in an instructed delay task. The SR nerve was stimulated with biphasic constant-current pulses (100 $\mu \mathrm{s} /$ phase $)$ at a constant frequency of $3 \mathrm{~Hz}$ and at $2 \times$ threshold, measured from the size of orthodromic volleys recorded in the proximal cuff electrode (Fig. 1C). As shown in Figure $1 D$, the area of the afferent volley was constant throughout the task in both flexion and extension trials. This result confirms that electrical stimulation applied through the distal cuff electrode activated SR afferents consistently even during dynamic hand movement.

For the cortical recording, electrode penetrations were made in precentral and postcentral cortex with a grid spacing of $1 \mathrm{~mm}$ (Monkey K) or
$2 \mathrm{~mm}$ (Monkey M). The electrode was advanced with a manual hydraulic microdrive and recording of cortical SEPs were performed at $0.5 \mathrm{~mm}$ intervals, starting from the depth that electrical activity of cortical neurons was first recorded. At each recording location, SEPs evoked by SR stimuli were compiled and averaged. The SEPs with maximal amplitude in each track were used as the representative SEP for each penetration. In monkey M (first monkey), the sensorimotor cortex, including premotor cortex and area 1, were mapped systematically. In monkey $\mathrm{K}$ (second monkey), recording was targeted to $\mathrm{M} 1, \mathrm{~S} 1$, and ventral PM using the movement evoked by intracortical microstimulation and the latency difference between responses in precentral and postcentral gyrus. The location of each area was confirmed histologically postmortem. For spinal recording, electrode penetrations were made to find single and/or multiunit activity evoked by SR stimuli. Once clear responses were recorded, the electrode was further advanced to the site of the largest amplitude SR-evoked potential. Again, the SEPs with maximal amplitude in each track were taken for analysis.

Measurement of SEPs. The SEPs evoked by SR stimulation were averaged separately for each behavioral epoch. The onset latencies and peak areas of SEPs were measured as illustrated in Figure $2 \mathrm{~A}$. The onset latency of extracellular field potentials was measured from the onset of the stimulus to the onset of the downward (negative) deflection of the earliest component of the field potential. The baseline was taken as the $20 \mathrm{~ms}$ of the record before the stimulus was delivered. The size of the SEPs was measured by the peak area under the baseline from the onset to the offset of the averaged waveform. SEPs in M1 showed small positive components before the negative component that probably reflect the remote recording of the large SEPs of neighboring area $3 \mathrm{~b}$ (Fig. 3). In this case, the latency was measured to offset of the positive component.

Measurement of movement kinematics. The angular position of the wrist joint was measured by the manipulandum and recorded along with other signals at a sampling rate of $1 \mathrm{KHz}$, and smoothed by moving averages (100 point). The derivative of smoothed position signal was also calculated to obtain speed measures. Averages of both signals were aligned with the onset of active and passive movement in flexion and extension trials and computed over an averaging window of $1.1 \mathrm{~s}(-100$ $\mathrm{ms}$, and $+1000 \mathrm{~ms}$ from each onset). Maximal or minimal value of the position derivative during the averaging window was defined as the peak speed of each trial. Differences between maximal or minimal angle were defined as the amplitude of each movement, and the average of the derivative signal was defined as a mean speed of each trial.

Simultaneous recording of spinal and cortical SEPs. In a preliminary attempt to evaluate the covariance of the sizes of SEPs across SC and cortical sites, we made simultaneous recordings from the three cortical areas and SC. Once the mapping of SEPs over S1, M1, and PM were completed, glass-coated tungsten electrodes were implanted at the site of the largest recorded SEPs in each area in monkey $\mathrm{M}$. The electrodes were implanted while recording the SR-evoked SEPs as the monkey performed the task. When the largest SEP was recorded in each track, the electrode and its connector were attached to the skull using dental acrylic. After this procedure, the craniotomy was completely covered by dental acrylic. Subsequently, the cervical recording chamber was implanted. This allowed us to simultaneously record SR-evoked SEPs in three cortical sites (via implanted electrode) and spinal cord (via movable electrode) (see Figs. 5, 9). Successful recording of the SEPs of SC, simultaneously with clean SEPs from three cortical areas, was made only from one SC site (in a single experiment). Therefore, the data obtained by the implanted electrodes was used only for the covariation analysis between the cortex and spinal cord (see Results, below), but not in the analyses of the latency, area, and task-dependent modulation of the SEPs.

Statistical tests. A one-way ANOVA with post hoc comparison (Tukey's test) was used for comparing the area and onset latency of SEPs among four recorded areas. A multiway ANOVA was used for comparing the SEPs of each monkey, behavioral epoch, recorded area, and movement direction. Dunnet's test was used for comparing the SEPs of multiple behavioral epochs relative to the rest. Finally, Student's $t$ test was used for pairwise comparison. The trial-by-trial covariation of the SEPs across four recording areas was evaluated by Pearson's correlation coefficient. 
Histological procedures. Near the conclusion of each experiment, we made small electrolytic lesions at several sites within the gray matter of cerebral cortex and spinal cord by passing a DC current $(30 \mu \mathrm{A}$ for $30 \mathrm{~s})$ through the recording electrodes. Animals were killed with pentobarbital sodium (50 $\mathrm{mg} / \mathrm{kg}$, i.v.) and perfused with $10 \%$ Formalin or $4 \%$ paraformaldehyde. The cortex and spinal cord were cut into $50 \mu \mathrm{m}$ sections and stained with cresyl violet.

\section{Results}

\section{Database}

In monkey $\mathrm{M}$, successful recordings were made from 69 cortical sites and significant SEPs were recorded from 46 sites. The onset latencies and the areas of SEPs of monkey $\mathrm{M}$ are illustrated in the cortical maps shown in Figure 2, $B$ and $C$. The onset latencies of SEPs (Fig. 2B) were obviously different for sites in precentral and postcentral gyrus. All of the postcentral SEPs had an onset latency $<9.5 \mathrm{~ms}$, while all of precentral latencies were longer than 9.5 ms. In addition, the size of SEP (Fig. 2C) was largest in the sites just anterior and posterior to the central sulcus (CS). These results suggest that onset latency of SRevoked SEPs can be used to localize the coordinates of the CS. To further document these measures in each division within the sensorimotor cortex (PM, M1, S1), we selected 25 sites in $\mathrm{PM}(n=8), \mathrm{M} 1(n=7), \mathrm{S} 1$ area $3 \mathrm{~b}(n=6)$, and $\mathrm{S} 1$ area $1(n=4)$ (Fig. $2 D)$. In Monkey $\mathrm{K}$, instead of mapping wide cortical areas (as shown in Fig. $2 B, C$ ), we targeted the equivalent cortical area that was selected in monkey M. Using the estimated coordinate of CS, we recorded SEPs from each area $(n=31: \mathrm{PM}=9, \mathrm{M} 1=11, \mathrm{~S} 1$ area $3 \mathrm{~b}=11$; Fig. $2 E$ ). SEPs from spinal cord were recorded in 29 sites (monkey $\mathrm{M}$ ) and eight sites (monkey K). In total, SEPs that were used for analysis were recorded from 52 intracortical sites ( 25 for monkey $\mathrm{M}$ and 31 for monkey $\mathrm{K}$ ) and 37 intraspinal sites ( 29 for $\mathrm{K}$ and 8 for $\mathrm{M}$ ).

\section{SR-evoked field potentials}

Figure $3 A$ shows typical examples of SEPs recorded in the four sites: SC, S1 (area 3b), M1, and PM. These SEPs were compiled and averaged over all behavioral epochs (Fig. 1B). A sharp negative deflection was characteristic of SEPs at all four sites. The amplitudes of SEPs were largest in S1 and smaller in M1, PM, and SC. The onset latency was shortest in SC (4.1 $\mathrm{ms})$, longer in S1 (7.1 ms), and longest in M1 (10.7 ms) and PM (11.2 ms) (Fig. 3B). The locations of the electrodes in these recording sites were histologically confirmed (Fig. 3C).

B

C
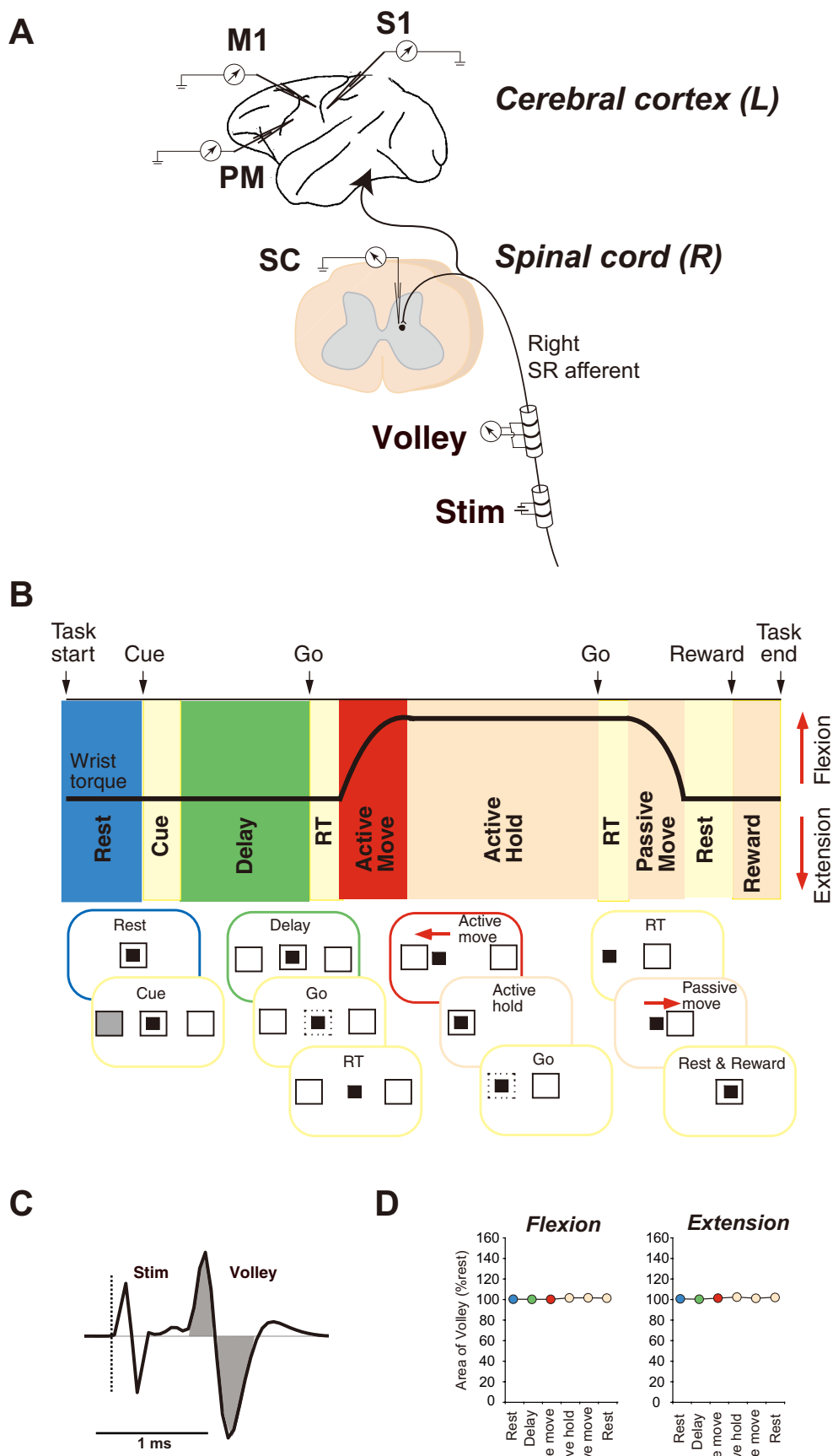

D
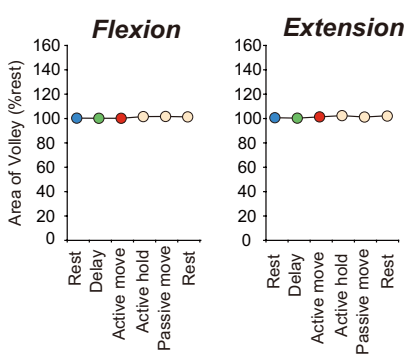

Figure 1. Methods. $\boldsymbol{A}$, Nerve stimulation. Two nerve cuffs were implanted on the SR of the right arm: a distal bipolar cuff for stimulation (Stim) and a proximal tripolar cufffor recording volleys. Constant stimulus frequency $(3 \mathrm{~Hz})$ and current $(2 \times$ threshold current, which activates the afferent fibers coding tactile stimuli) were used throughout the recording period. SR-evoked field potentials were recorded from both cervical spinal cord and cerebral cortex. Spinal SEPs were recorded within the gray matter of ipsilateral spinal cord and cortical SEPs were recorded in contralateral M1, PM, and S1 of wrist-arm representation. $\boldsymbol{B}$, Behavioral task. Typical torque trace during a single flexion trial is shown with task epochs. Diagrams below depict the cursor controlled by the monkey (small filled square) and targets (larger squares) on video screen for the 10 epochs: first Rest, Cue, Delay, first RT, Active Move, Active Hold, second RT, Passive Move, second Rest, and Reward (for details, see Materials and Methods). C, Average of orthodromic volleys $(n=21,990)$ recorded in the proximal cuff electrodes. Gray shading represents the area of volleys measured for comparing them across the behavioral epochs. D, Area of orthodromic volley during behavioral task shown in $\boldsymbol{B}$ (22 flexion trials, 22 extension trials). Volleys were averaged separately for each behavioral epoch and areas plotted as percentage of rest.

These results were consistently observed in both monkeys (Fig. 4). The average onset latency (Fig. 4, top) and peak area (Fig. 4 , bottom) of SEPs in each area are shown separately for monkey $\mathrm{M}$ (Fig. 4A) and monkey K (Fig. 4B). In both animals, analysis of 
Onset latency Peak area

A

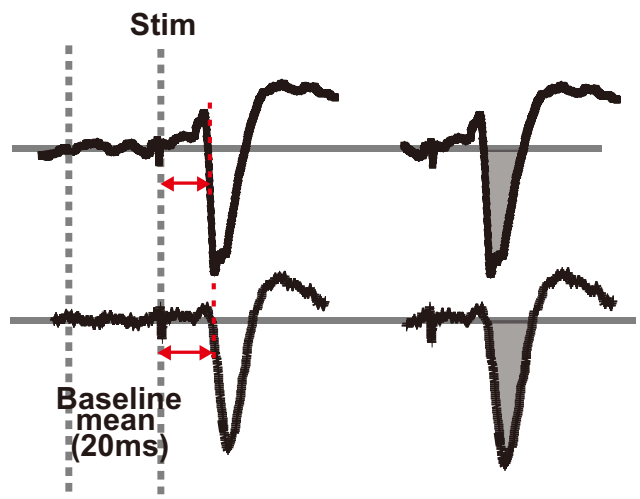

B

Onset latency (Monkey M)

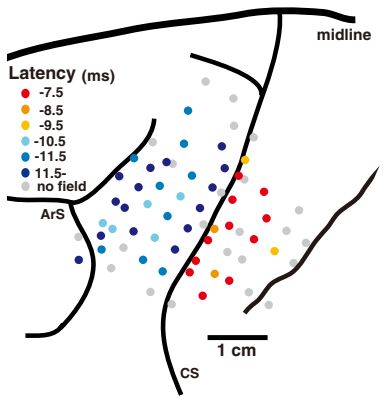

C

Peak area (Monkey M)

D

Representative cortical sites (Monkey M)
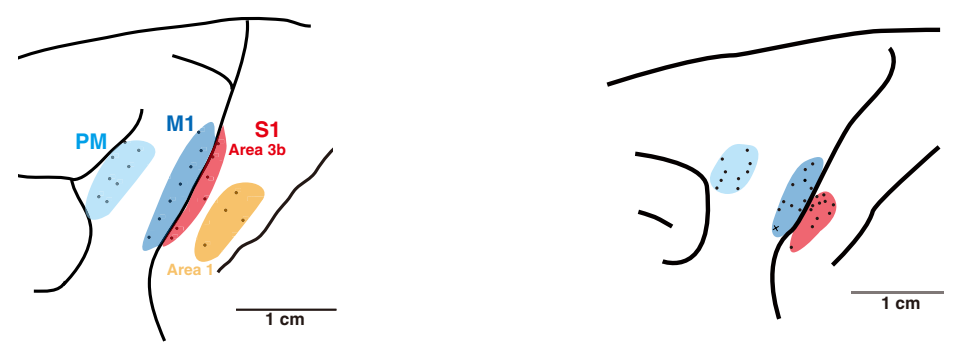

Figure 2. Measurement of SEPS. A, Measurement of onset latency (red arrow) and peak area (gray shading) of the SEPs. Stim, Time of stimulation of SR. Baselines (horizontal lines) were determined from mean of the background activity ( $20 \mathrm{~ms}$ before stimulation). Top, SEPs with preceding positive component (M1). Bottom, SEPs without preceding positive component. $B, C$, Penetration map of monkey M shown with onset latency $(\boldsymbol{B})$ or the peak area (C). Largest SEPs in each penetration were analyzed. The latency and area was color-coded. $\boldsymbol{D}, \boldsymbol{E}$, Site in the cortex of monkey $\mathrm{M}(\boldsymbol{D})$ or monkey $\mathrm{K}(\boldsymbol{E})$ that were subjected to further comparison of the latency, area, and task-dependent modulation. Note that area 1 of $\$ 1$ was not recorded in monkey $K$ and data of monkey $M$ were not subjected to the analysis of task-dependent modulation. " $x$ " in $E$ is the site where thumb extension movement was evoked by electrical stimulation with intracortical microstimulation ( $10 \mu \mathrm{A}, 0.1 \mathrm{~ms}$ biphasic pulses, 10 pulses at $333 \mathrm{~Hz}$ ).

latencies in both monkeys (Tukey's test, $p<0.05)$. These data indicate that the input from SR arrived earliest in the SC $[5.6 \pm 1.5 \mathrm{~ms}$ (means $\pm \mathrm{SD}$; monkey $\mathrm{K})$; $4.7 \pm 0.6 \mathrm{~ms}$ (monkey $\mathrm{M})$ ], and earlier in S1 [area 3b: $7.9 \pm 0.9 \mathrm{~ms}$ (monkey $\mathrm{K}$ ), $7.0 \pm 0.1 \mathrm{~ms}$ (monkey M); area 1: $7.6 \pm$ $0.6 \mathrm{~ms}$ (monkey M)] than M1 [10.8 \pm 0.9 ms (monkey K), $12.4 \pm 1.4 \mathrm{~ms}$ (monkey $\mathrm{M})$ ] or PM [11.35 $\pm 0.5 \mathrm{~ms}$ (monkey $\mathrm{K})$, $11.57 \pm 0.3 \mathrm{~ms}$ (monkey M)]. Differences of onset latency between $\mathrm{S} 1$ and the motor areas could reflect the time required for intracortical transmission from postcentral to precentral gyrus (Ghosh and Porter, 1988; Aizawa and Tanji, 1994). Within motor cortices, we found a significant difference between M1 and PM in monkey M (Tukey's test, $p<0.05$ ) but not in monkey K. Within sensory cortices, we found no significant difference between area $3 \mathrm{~b}$ and area 1 in monkey $\mathrm{M}$ (no comparison was available for monkey K).

Post hoc comparison on the area of SEP revealed a significant difference between SC and S1 (area 3b) in both monkeys (Tukey's test, $p<0.05$ ). Within motor cortices, we found a significant difference between $\mathrm{M} 1$ and PM in monkey $M$ (Tukey's test, $p<0.05$ ) but not in monkey $\mathrm{K}$. Within sensory cortices, we found no difference between area 1 and $3 b$ in monkey M (no comparison was available for monkey K).

\section{Modulation of evoked SEPs during task epochs}

Our primary objective was to determine whether the size of SEPs in each area of cortex and spinal cord was modulated as a function of the behavioral epochs. Figure 5 shows nonaveraged SEPs as well as EMGs and wrist kinematics during a single flexion trial (Fig. 5A). The superimposed examples (Fig. 5B) show robust (S1, PM, M1) or modest (SC) suppression of the size of SEPs during active movement. In addition, the evoked responses were reduced during the instructed delay period in M1 and PM. In this example, SEPs of each area were recorded simultaneously, but the following analyses were made on the SEPs that were recorded indepen-

onset latency and peak area was performed with a one-way ANOVA contrasting four factors for recording sites. A significant main effect of recording sites emerged in both the onset latency $\left[\right.$ monkey $\mathrm{K}: F_{(3,33)}=57.80$, mean square error $(\mathrm{MSE})=55.17$, $p<0.001$; monkey M: $\left.F_{(4,47)}=237.73, \mathrm{MSE}=122.66, p<0.001\right]$ and peak area (monkey K: $F_{(3,33)}=3.605, \mathrm{MSE}=110.55, p<$ 0.023; monkey M: $\left.F_{(4,47)}=9.32, \mathrm{MSE}=25,259.3, p<0.0001\right)$.

Post hoc comparison of the onset latency revealed significant differences between SC and S1 in both monkeys (Tukey's test, $p<0.05)$. Among the cortical areas, SEPs in S1 had shortest dently from each area using movable electrodes. To quantify these effects, SEPs recorded at all cortical $(n=52$; Fig. $2 D)$ and spinal $(n=37)$ sites were compiled and averaged separately for each of the behavioral epochs, movement direction (flexion or extension), monkey, and recorded area. Result of multiway ANOVA suggested that there were significant effects $\left(F_{(11,1178)}=\right.$ $6.72, \mathrm{MSE}=33,295.9, p<0.0001)$ of both area $(F=5.68, p=$ $0.0007)$ and behavioral epoch $(F=8.50, p<0.0001)$, but not monkey $(F=0.50, p=0.47)$ or movement direction $(F=0.52$, 
$p=0.46)$. Therefore, we combined the SEP areas for both monkeys and those for flexion and extension trials and present resultant data for each area (Figs. 5C, 6C). As clearly shown, the size of SEP in all recorded areas was suppressed during the task. Next, we made a detailed analysis for each behavioral epoch.

Figure 6 compares the averaged SEPs for the instructed delay and active movement relative to the control (Rest) in each area. Representative examples (one site for each recorded area) are shown in Figure $6 \mathrm{~A}$. The most obvious modulation of SEPs in all recorded areas was the suppression during active movement. Suppression of SEPs was also clear during the instructed delay period in M1 and PM, but not in S1 or SC. Next, we measured the area of SEPs of these representative sites and plotted them relative to the movement onset (extension) by moving average $($ window $=500 \mathrm{~ms}$, step $=100$ ms; Fig. $6 B$ ). In these plots, two types of suppression were detectable. First, the area of SEPs after movement onset (starting at time 0 ) was smaller than that of rest in all recorded areas (Dunnet's test, $p<$ 0.05). Second, the area of SEPs before movement onset (even $800 \mathrm{~ms}$ before the onset of movement) was consistently smaller in M1 (Dunnet's test, $p<0.05$ ). The SEPs in PM showed similar characteristics as M1 but the amount of the suppression did not reach statistical significance. Figure $6 C$ shows the average size of SEPs recorded at all cortical ( $n=$ $52)$ and spinal sites $(n=37)$ in these three behavioral epochs. Again, the two types of suppression shown in Figure 6, $A$ and $B$, were confirmed in this population analysis. First, during active movement, the size of SEP was significantly suppressed in all three cortices and spinal cord ( $t$ test, $p<$ 0.05). This widespread reduction during active movement suggests a general type of suppression. Second, during the instructed delay period, the amplitudes of the SEPs were smaller than those of rest in $\mathrm{M} 1$ and $\mathrm{PM}$ ( $t$ test, $p<0.05$ ), suggesting a more specific suppression of SEPs during the instructed delay period.

In addition to dynamic movements, we also examined changes in SEPs during the static hold. Figure $7 A$ compares the sizes of SEPs between rest and active static hold (for flexion and extension). Similar to the suppression during active movement (Fig. 6), suppression during active hold was clearly present in all recorded regions for both flexion torque [paired $t$ test, $p=0.03$ (SC), $p=0.0002$ (S1), $p=$ $0.0002(\mathrm{M} 1)$, and $p<0.0001(\mathrm{PM})$ ] and extension torque [paired $t$ test, $p=0.0019$ (SC), $p=0.0001$ (S1), $p<0.0001$ (M1), and $p<$ $0.0001(\mathrm{PM})]$. This suggests that the suppression during a static motor output could be a common phenomenon in the CNS.
C
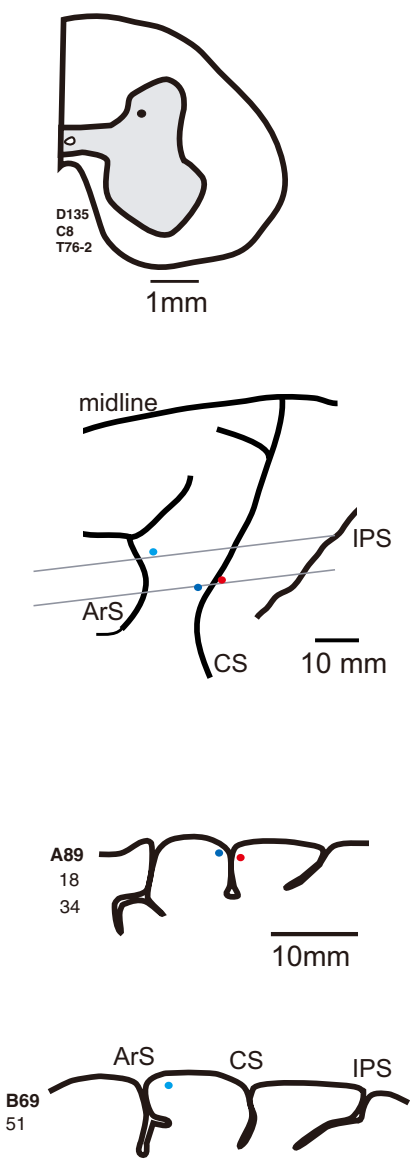

Figure 3. Example of SEPs. A, Typical examples of SR-evoked field potentials (averaged over all task epochs) in M1, S1, PM, and SC. Most recording sites had predominantly negative field potentials. This negative wave was followed by a positive component in S1 (red), and was sometimes preceded by a small positive component in M1 (dark blue) and PM (light blue). The negative field potentials probably reflect synaptic potentials in local neurons; the positive components could reflect recording of remote field potentials generated in adjacent regions. We analyzed the first negative component. $\boldsymbol{B}$, Fields shown superimposed in faster sweeps. Triangles mark onset of the negative component. $C$, Locations of histologically confirmed sites where the recording shown in $\boldsymbol{A}$ and $\boldsymbol{B}$ was made. First, Transverse section of cervical spinal cord at C8 level. Second, Top view of cerebral cortex (left hemisphere). Third and fourth, Saggital sections made from the level shown in the top view (horizontal lines). Recordings from monkey M. ArS, Arcuate sulcus; IPS, intraparietal sulcus.

Fig. $7 B$ compares the size of SEPs between active and passive movements. Little difference was observed between active flexion and passive flexion in the recorded regions $[t$ test, $p=0.38$ (SC), $p=0.03$ (S1), $p=0.80$ (M1), and $p=0.29$ (PM); Fig. 7B, left], although suppression during active movement tended to be greater. This observation confirms previous reports by Chapman and colleagues (Chapman et al., 1988; Jiang et al., 1990). In contrast, suppression was significantly greater during active extension than during passive extension [ $t$ test, $p=0.04$ (SC), $p=$ $0.0002(\mathrm{~S} 1), p=0.04$ (M1), and $p=0.0002$ (PM); Fig. 7B, right]. These results could be explained by two potential mechanisms that induce movement-related suppression. First, the size of sup- 
A
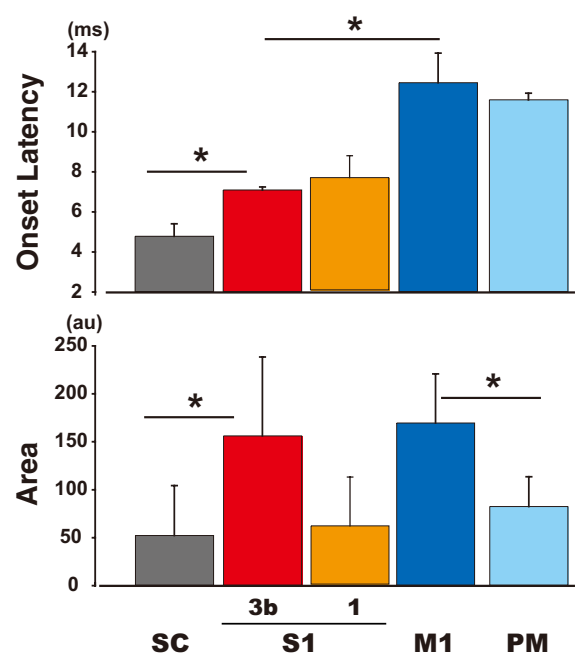

B

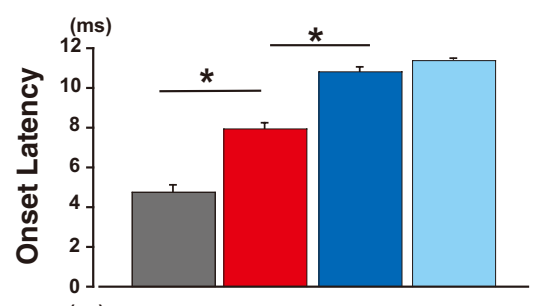

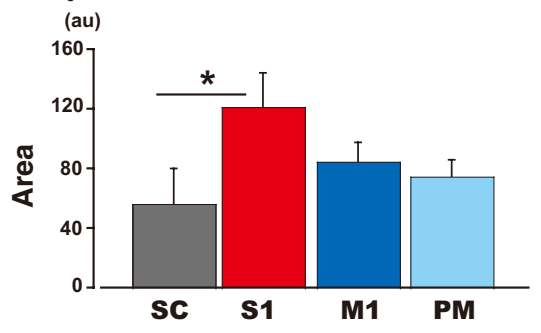

Figure 4. Onset latency and area of SEPs. $\boldsymbol{A}, \boldsymbol{B}$, Means ( \pm SD) of the onset latencies and areas of SR-evoked field potentials in monkeys $\mathrm{M}(\boldsymbol{A})$ and $\mathrm{K}(\boldsymbol{B})$. Individual recording sites for PM ( $n=8$ and 9$), \mathrm{M} 1$ ( $n=7$ and 11$)$, and $\mathrm{S1}(n=6$ and 11$)$ are shown in Figure 2, $D$ and $E$. Intraspinal recording sites $(n=29$ and 8 ) are not shown. Onset latencies were shorter in SC and S1 than M1 and PM. ${ }^{*} p<0.01$ (Tukey's test).

pression is influenced by movement kinematics (Angel and Malenka, 1982), so the difference between active and passive extension could be attributed to either the size or speed of movement. To test this possibility, the position signals of the manipulandum (angle of wrist; Fig. $5 A$ ) were averaged aligned with onset and offset of all flexion $(n=2710)$ and extension $(n=2594)$ trials used for the analysis shown in Figure $7 B$. Subsequently, we calculated the amplitude, mean, and peak speed of movements from individual traces and compiled them for four movement conditions (Fig. 7C, data from two monkeys combined). A significant main effect of movement condition emerged in the amplitude $\left(F_{(3,10578)}=974.0\right.$, MSE $=11,716.1, p<0.000)$, mean speed $\left(F_{(3,10551)}=223.7\right.$, MSE $=35,086.5, p<0.0001)$ and peak speed $\left(F_{(3,10579)}=\right.$ 447.3, MSE $=734,121, p<0.0001)$. Post hoc comparison revealed that all comparisons among each movement condition in the three kinematic parameters were significantly different (Tukey's test, $p<0.05$ ). This result may suggest that the kinematics could affect the suppression of SEPs, but it is not clear whether they could explain the difference in the size of SEPs between active and passive movement (Fig. $7 B$ ) in any systematic way. For example, passive movements were significantly smaller and slower than active movements (in both directions of movement), but the difference of the area of SEPs cannot be explained by these difference in the kinematic parameter since the difference in SEPs were found only in the extension. Future studies to resolve the effect of movement kinematics on the suppression of spinal and cortical SEPs would need to use tasks specifically designed to separate size and speed of movements.

Second, we previously reported that cutaneous afferent input to spinal cord was suppressed during active movement by presynaptic inhibition, and that inhibition could be induced by descending motor command as well as self-induced reafference (Seki et al., 2003), and that presynaptic inhibition was stronger during active extension than flexion (Seki et al., 2009; their Fig. 9). This evidence suggests that the larger suppression in active relative to passive extension could be induced in part by presyn- (cf. Fig. 8C).

\section{Relation between SEP modulation and motor performance}

Unlike the suppression of SEPs during active extension, which could be induced by either bottom-up or top-down mechanisms, modulation of SEPs during the instructed delay period could not be caused by bottom-up reafferent mechanisms, since there was no movement during this period. Therefore, the suppression of SEPs found in M1 and PM during the instructed delay period (Fig. 6) would be induced by a motor-related command (topdown). We explored the functional relevance of this suppression by comparing the size of suppression with the reaction times of subsequent movements.

We first sorted all successful trials by their reaction times and obtained a median of the reaction times, and compiled SEPs evoked during delay periods associated with short (shorter than median) and long (longer than median) reaction times. Figure 8, $A$ and $B$, show representative examples of two flexion and two extension trials, with shorter and longer reaction times. As shown, for the extension movements, SEPs were more strongly suppressed before the shorter reaction times. Similar analyses performed on the SEPs from motor cortex (data from M1 and PM were pooled) in two monkeys are summarized in Figure $8 C$. Data for M1 and PM in monkey $\mathrm{M}[n=12(\mathrm{M} 1=5, \mathrm{PM}=7)]$ and monkey $\mathrm{K}[n=14(\mathrm{M} 1=7, \mathrm{PM}=7)]$ were similar and pooled $(n=26)$. SR-induced SEPs during the instructed delay period before extension movement with shorter reaction times were significantly smaller than that with longer reaction times in extension trials (paired $t$ test, $p=0.01, \mathrm{df}=25$ ), but this was not seen for flexion trials (paired $t$ test, $p=0.679, \mathrm{df}=25$ ). These results suggest that suppression of sensory input from SR to motor cortex is significantly correlated with preparation and execution of rapid wrist extension.

Our behavioral task was designed to discourage any movement during the instructed delay period, and we confirmed that there was negligible EMG during this period (Fig. 5A). However, it is impossible to reject the possibility of a very small amount of muscle activities just before the go signal, and one can argue this 
A

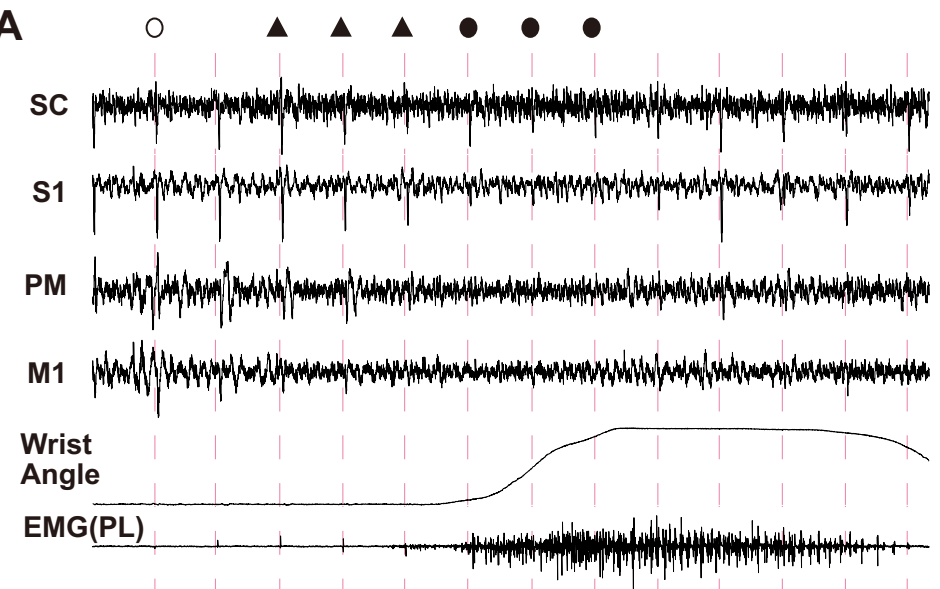

SR

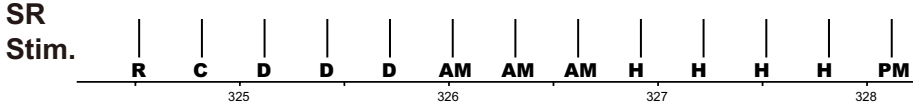

Time (sec)

B

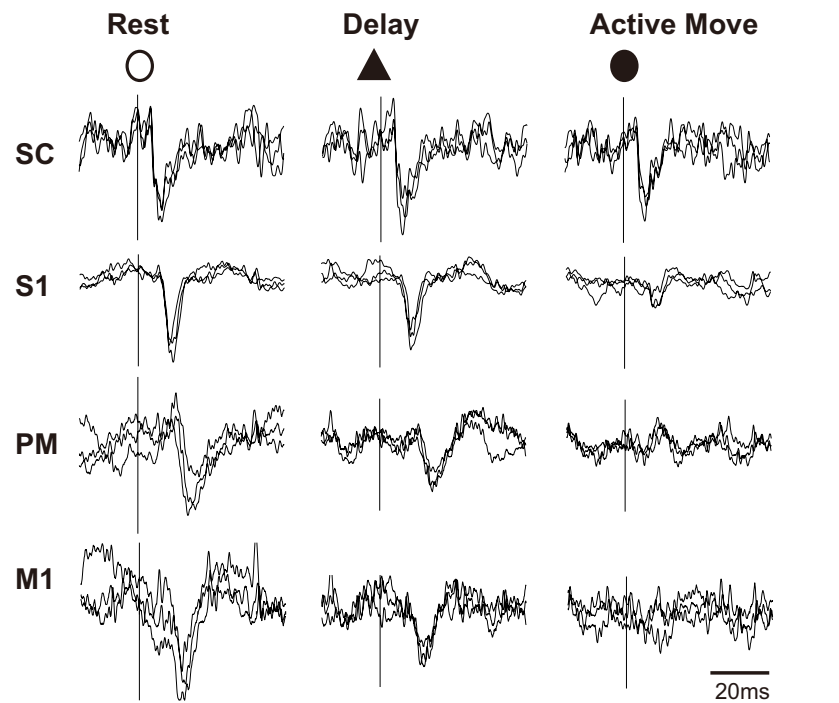

C

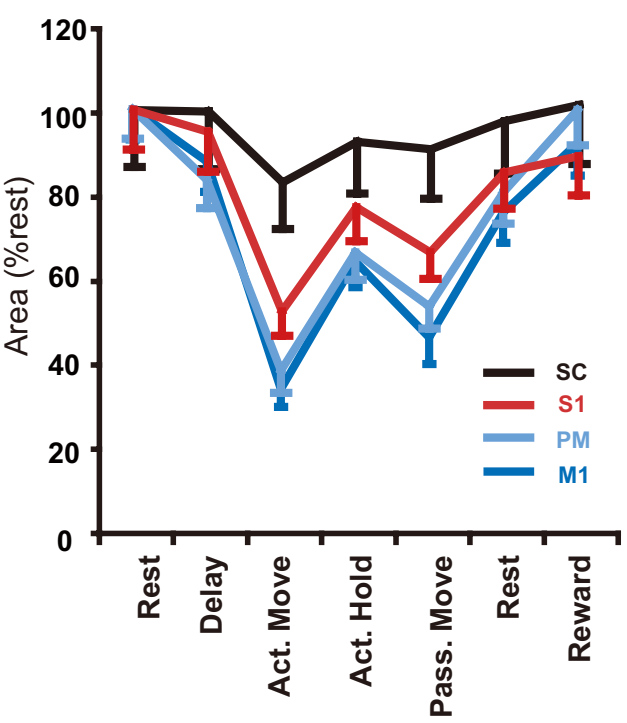

Figure 5. Modulation of SEPs during task epochs. $\boldsymbol{A}$, Example of a recording in a single trial (flexion). Field potentials were recorded simultaneously in the spinal cord and in the cerebral cortex (S1, PM, M1). Dashed lines indicate the timing of SR stimuli (Stim.) shown at bottom. PL, Palmaris longus; $R$, rest; $C$, cue; D, instructed delay; AM, active movement; $H$, hold; PM, passive activity may induce reafference that could suppress SEPs. To test this possibility, we compared the size of SEPs in the earlier and later part of the instructed delay period, using the same dataset as Figure 8 , $A-C$. As shown in Figure $8, D-F$, we found no difference in the size of SEPs of motor cortices in both flexion (paired $t$ test, $p=$ $0.83, \mathrm{df}=25$ ) and extension (paired $t$ test, $p=0.65, \mathrm{df}=25)$. This result supports the hypothesis that the suppression of SEPs occurring during the instructed delay period was not induced by any bottom-up reafference signal.

\section{Modulation of covariation of SEPs} among cortical areas and spinal cord In the above results, the task-dependent modulation of SEPs in spinal cord and cortices (Figs. 5-8) was analyzed using data recorded from each area separately. This allowed us to document the largest SEPs in each recording track and compare the behavior of maximal responses in each area. To obtain additional information on the potential sources of modulation, we also performed an experiment using simultaneous recordings at representative sites to document the trial-by-trial covariation of the SEPs in these areas. Figure 9 illustrates the results (see also Fig. 5A, $B$ ). The magnitude of suppression of SEPs in two connected regions (e.g., M1 and PM) would be expected to covary if they were modulated by a common mechanism. To gain relevant evidence, we recorded simultaneous SEPs from one electrode each in S1, M1, PM (implanted), and SC (moveable microelectrode). The task-dependent modulation of these SEPs is shown in Figure 9A. We then calculated the correlation coefficient for pairs of SEPs in individual trials of each behavioral epoch. Figure $9 B$ shows the taskdependent covariance between each of the cortical and spinal SEPs. The overall modulation of covariance (evaluated by difference between the maximal and minimal $r$ value during task: $\Delta r$ ) was largest between SC and S1 $(\Delta r=0.60), \mathrm{S} 1$ and M1 $(\Delta r=$ $0.65)$, and M1 and PM $(\Delta r=0.51)$. The task-dependent modulation of covariance could be grouped into two types: transient

$$
\leftarrow
$$

movement. $\boldsymbol{B}$, Three superimposed SR-evoked field potentials during rest (open circle), instructed delay (triangle), and active movement (filled circle). Note reduction of SEP during active movement. $\boldsymbol{C}$, Epoch-dependent modulation of the area of SEPs evoked in M1, S1, PM, and SC. Data obtained in two monkeys (M1: $n=10$ and 6, PM: $n=9$ and $8,51: n=11$ and 10 , $S C: n=6$ and $25 ;$ Fig. $2 D, E)$; data in both flexion and extension trials were pooled and averaged. 


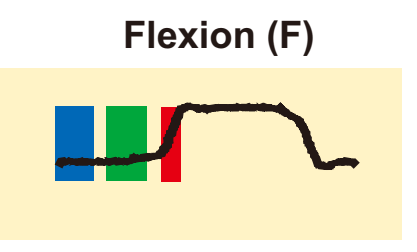

A

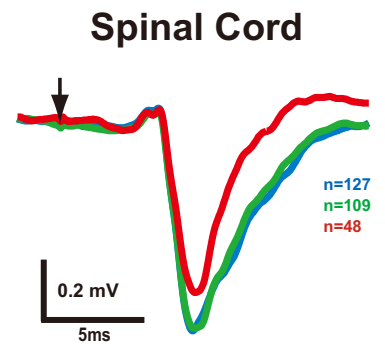

S1 (3b)

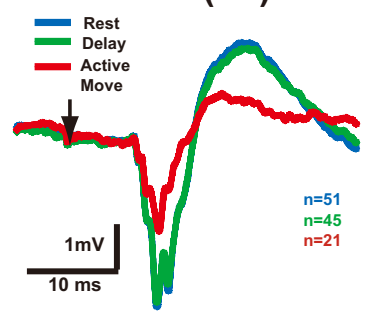

M1

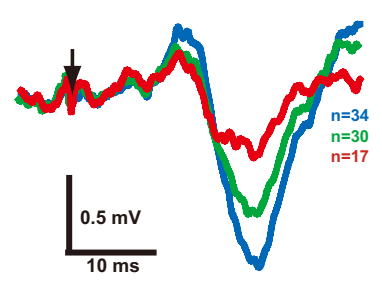

PM

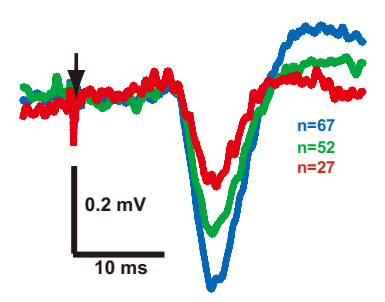

C

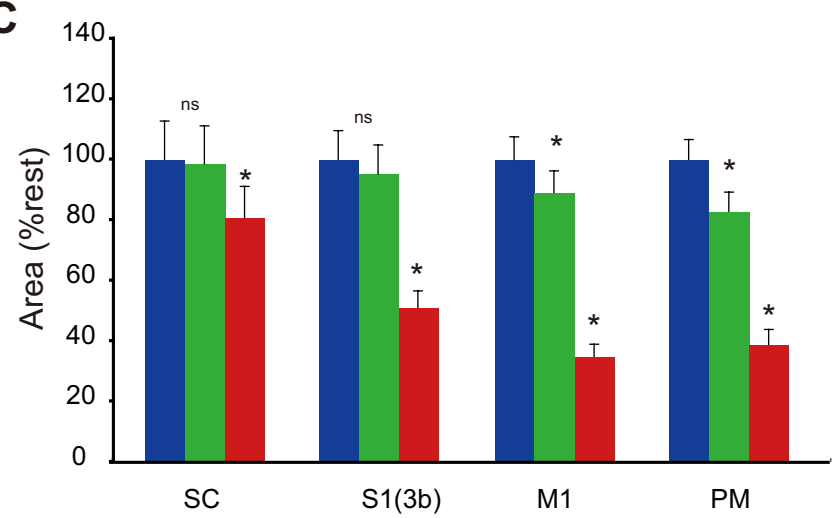

Extension (E)

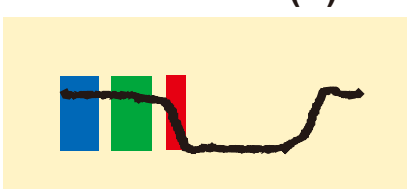

B

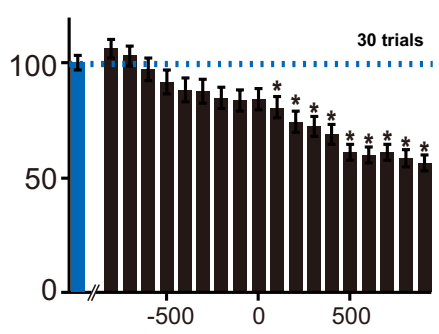

11 trials
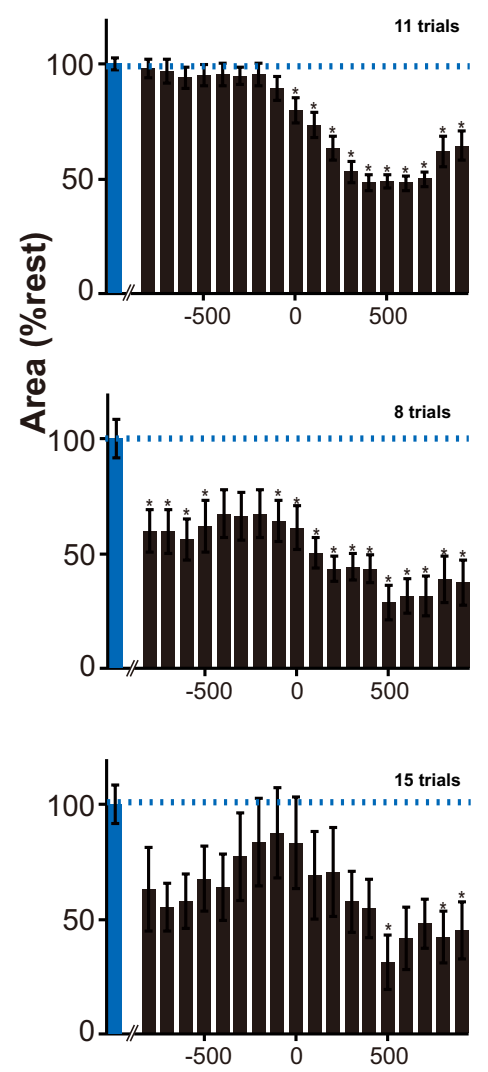

Time to movement onset (ms)

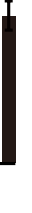


at multiple sites during preparatory periods as well as active and passive movements. We documented SEPs, which are generated by the postsynaptic potentials of cells in the vicinity of the recording electrodes. The magnitudes of the SEPs reflect both the size of the arriving synaptic input as well as the responsiveness of the postsynaptic cells to this input. Changes in responsiveness that could lead to decreased SEPs include postsynaptic inhibition of local neurons from topdown gating mechanisms or bottom-up collateral inhibition (Eccles et al., 1963; Ghez and Pisa, 1972; Seki et al., 2003, 2009). The data suggest the existence of top-down and bottom-up gating mechanisms operating at all levels during active movements, and top-down gating mechanism at motor cortex during preparatory periods.

\section{Stimulation methods}

The responses of cortical neurons to peripheral stimulation have been documented in many studies. For example, the somatotopic organization of postcentral cortex has been revealed by mapping responses to natural stimuli applied to a variety of peripheral regions and receptors (Mountcastle, 1957). While ideal for elucidating effective stimulus features, natural stimulation has limited value for investigating other issues. Since individual receptors are stimulated asynchronously in most forms of natural stimulation, it is difficult to define a precise latency of responses evoked by afferent input. Moreover, it is often hard to stimulate a given modality of receptor selectively. More importantly, the stimulus intensity cannot be accurately controlled during movement of the stimulated limb. By using electrical stimulation via an implanted cuff electrode we are able to stimulate the peripheral afferents synchronously and measure the latency of cortical and spinal-evoked responses (Figs. 1-4), stimulate exclusively cutaneous (but not muscle) afferents, and deliver constant stimulus intensities and afferent volleys even during dynamic behavior (Fig. $1 D$ ). These features allowed us to examine the modulation of peripherally induced response during volitional movement.

In this study, SEPs were recorded throughout task performance by stimulating at $3 \mathrm{~Hz}$. It is known that higher repetition frequency can suppress the amplitude of SEPs (Curtis and Eccles, 1960; Hultborn et al., 1996). Therefore, the size of SEPs documented in this study may have underestimated the sizes evoked by lower frequency stimulation. However, this stimulus frequency would not affect the relative size of suppression during task epochs, since it was constant throughout the task.

\section{Gating of serial transmission in ascending pathways during} active movement

The relative onset latencies of the SEPs are consistent with serial transmission of afferent activity from spinal cord to S1 to motor

C
Flexion
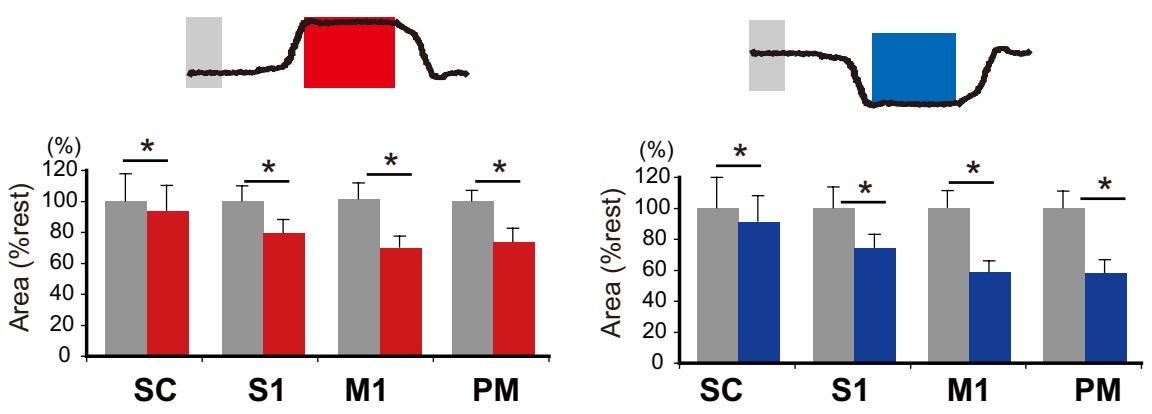

\section{Extension}

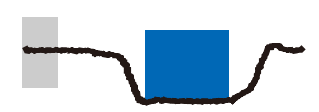

B

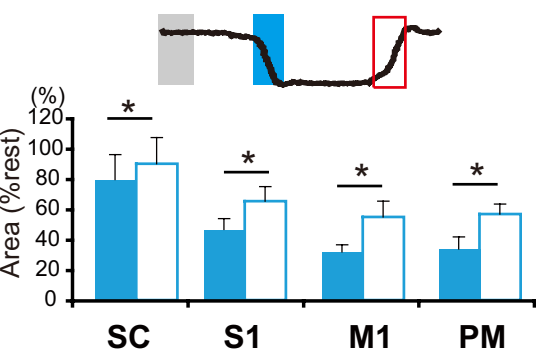

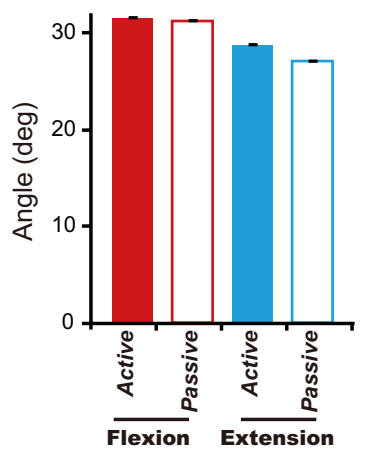
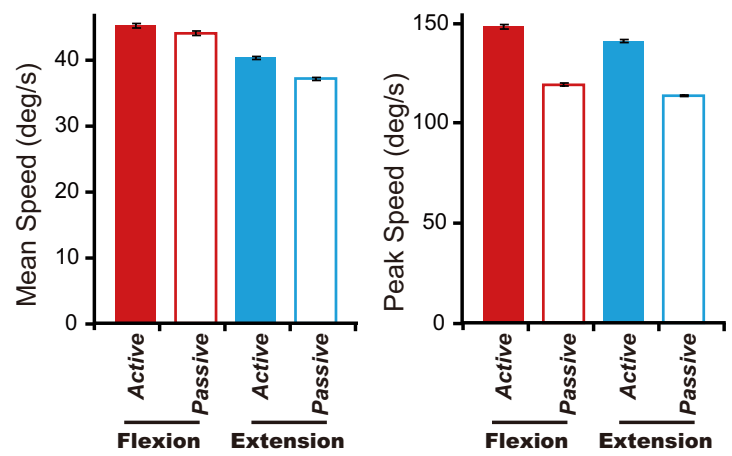

Figure 7. Modulation of SEPs during other behavioral periods. $A$, Comparison of the areas of SEPs between rest and sustained rce period. SEPs of most regions were suppressed during sustained flexion and extension. ${ }^{*} p<0.05$ (paired $t$ test). $\boldsymbol{B}$, Compar(bexsive flexion (red) and extension (blue). ${ }^{*} p<0.05$ ( $t$ test). Active extension (filled blue bar) induced differences within each kinematic parameter were significant ( $p<0.01$, Tukey's test).

areas (Fig. 4). Physical proximity to the periphery can obviously explain the earliest latency of SEPs $(4 \mathrm{~ms})$ recorded in SCs (Seki and Fetz, 2010), and responses in S1 (7-8 ms) probably represent the earliest cortical responses induced via the lemniscal pathway. The latency in M1 and PM was longer (10-11 $\mathrm{ms})$. The difference in the onset latency between S1 to M1 or $\mathrm{PM}$ is consistent with the latency recorded for orthodromic transmission from S1 to M1 (Aizawa and Tanji, 1994). Although a direct pathway from thalamus to motor areas has been reported (Lemon and van der Burg, 1979; Tracey et al., 1980), we will assume the SEPs recorded in M1 and PM were largely mediated by corticocortical connections from S1, and we will discuss the consequent implications for gating.

The largest and most widespread suppression of SR-evoked SEPs occurred during active movement, for both flexion and extension (Fig. 5C). Some of this widespread suppression is at- 
A

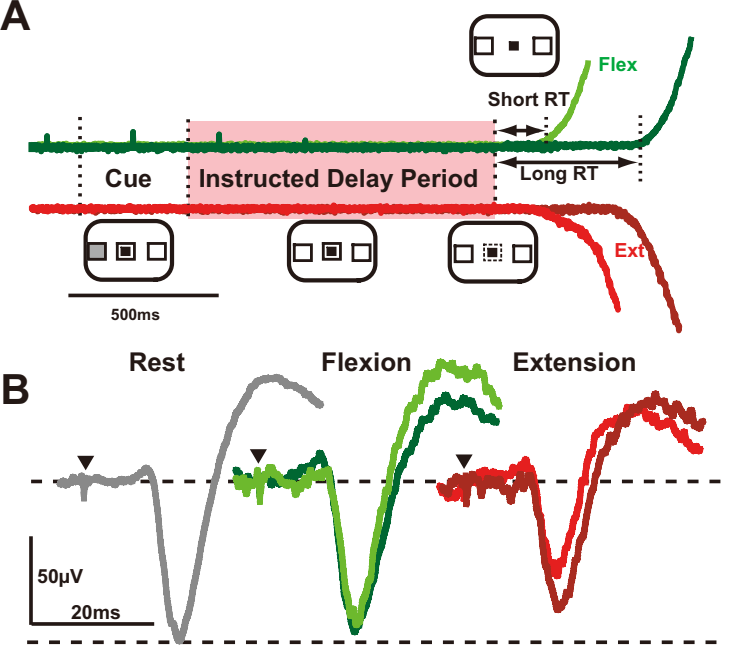

D

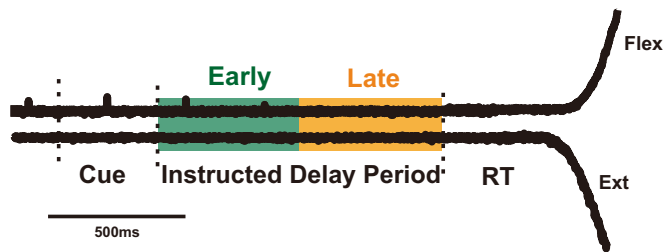

E

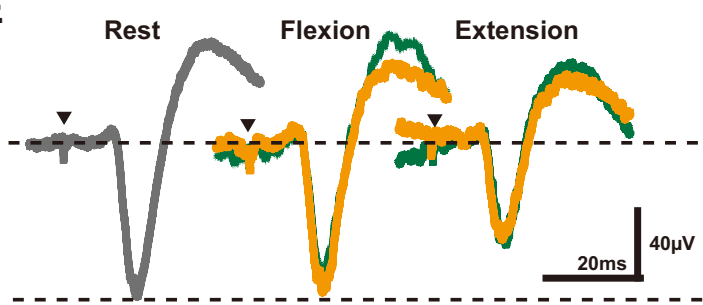

C

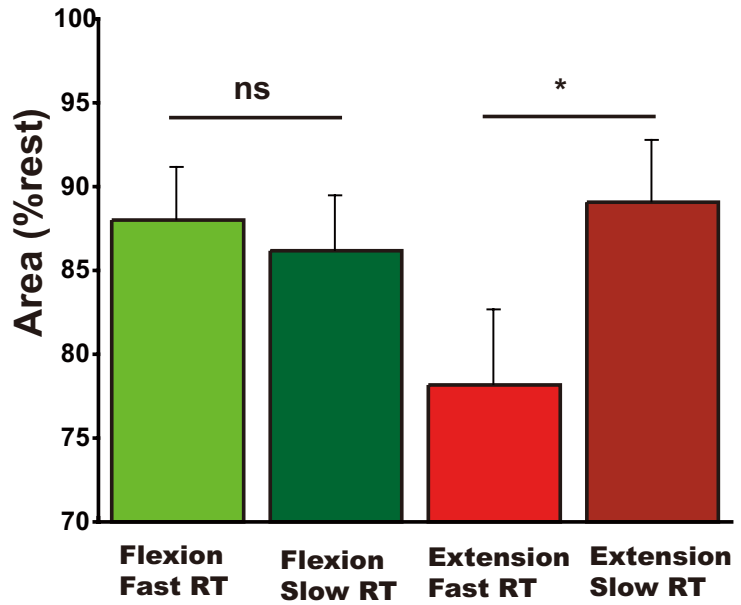

$\mathbf{F}$

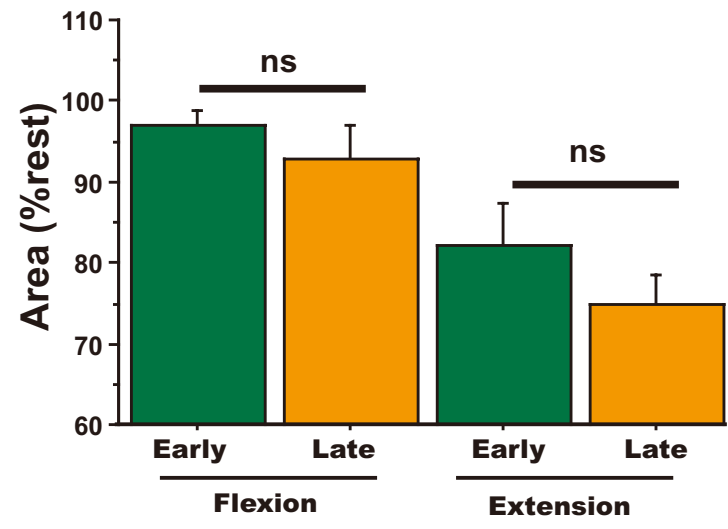

Figure 8. Relation between reaction times and SEP size during delay period. $\boldsymbol{A}$, Torque traces for flexion (green) and extension (red) trials with short or long reaction times. Reaction times were measured from the go signal to the onset of flexion or extension torque. Average traces aligned on go signal were compiled separately for reaction times longer and shorter than the median. $\boldsymbol{B}$, SR-evoked SEPs were compiled and averaged separately for stimuli applied during the instructed delay period before movements with fast and slow reaction times. Traces show examples of SEP in PM of monkey M. C, Means ( \pm SD) of the area of SR-evoked SEPs evoked during the instructed delay periods before flexion trials with faster (light green) and slower (dark green) reaction time and extension trials with faster (red) and slower (brown) reaction time. D, Torque traces for flexion and extension trials. "Early" and "Late" represent the first and second half of the instructed delay period. $\boldsymbol{E}$, SR-evoked SEPs were compiled and averaged separately for stimuli applied during early and late half of instructed delay period using same data as in $\boldsymbol{A}-\boldsymbol{C}$. $\boldsymbol{F}$, Means ( \pm SD) of the areas of SR-evoked SEPs evoked during early (green) or late (orange) part of instructed delay period. ${ }^{*} p<0.05$; ns, not significant $(p \geq 0.05)$ (paired $t$ test).

tributable to inhibition at the first spinal relay. Previous work showed that responses of first-order spinal interneurons to SR input are reduced during active movement, and that this reduction is produced at least in part by presynaptic inhibition of SR afferents, as demonstrated by measuring primary afferent depolarization (Seki et al., 2003, 2009). The projections from these inhibited interneurons could include neurons involved in the lemniscal pathway as well as spinothalamic cells (Seki et al., 2003), and this suppression at the initial relays would result in nonspecific reduction of SEPs throughout the lemniscal pathway, including thalamus and sensorimotor cortex (Chapman et al., 1988).

Our observations suggest that this widespread suppression could involve both top-down and bottom-up mechanisms. Suppression at spinal relays could be produced by a top-down gating mechanism accompanying commands to initiate movement, since it begins before onset of EMG activity (Seki et al., $2003,2009)$. In addition, some of the reduction of SEPs after onset of movement could also be due to bottom-up collateral inhibition partially induced by reafferent activity of the SR itself (Fig. 7). The amount of suppression during task performance (including active and passive movement and active hold period) relative to rest is substantially greater in S1 than in SC, and greater yet in motor cortical areas (Figs. 5-7). This might suggest the presence of mechanisms at these higher levels in addition to effects attributable to reduction in firstorder transmission.

\section{Gating of corticocortical transmission during the instructed} delay period

During the instructed delay period, the SR-evoked SEPs were significantly suppressed in M1 and PM but not in SC or S1 (Fig. 6). If afferent input is transmitted serially from $S 1$ to $M 1$, the greater reduction of SEPs in motor areas suggests that corticocortical transmission may be locally gated, perhaps via top-down signals from higher-order sites generating prepara- 
A

B

Flexion Extension
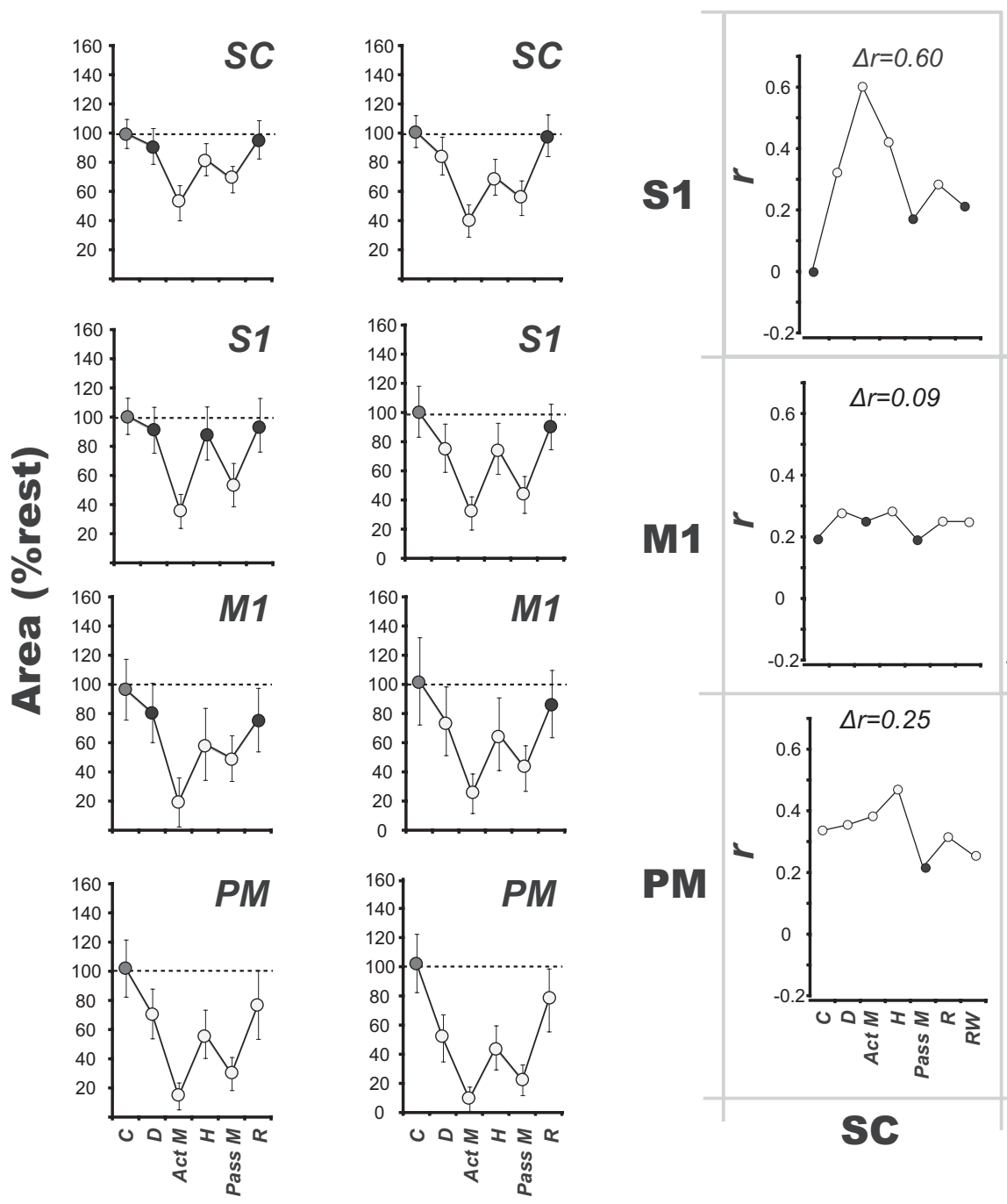

C
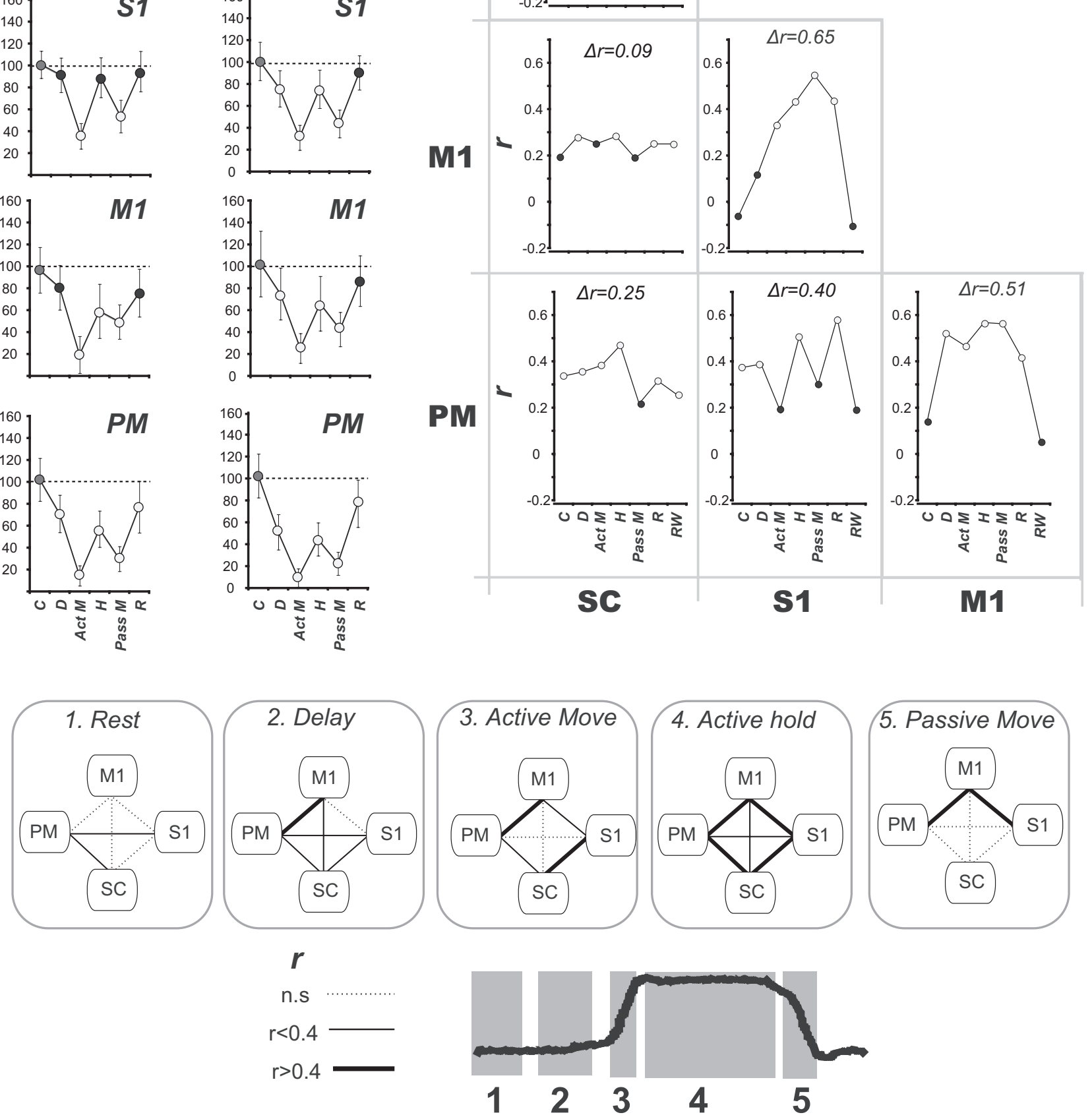

Figure 9. Task-dependent correlations between SEPS.A,Amplitudes of SEPs during flexion (left) and extension (right) trials as a function of task. Areas of SEPs in SC, S1, M1, and PM (as percentage of rest) were averaged separately fortaskepochs. Open circles indicate a significant differencefrom rest $(p<0.05)$. B, Plots of the correlation coefficientsforpairs of simultaneoustrial-by-trialSEPs, as afunction of task. Datafrom flexion and extension trials were combined. Correlations are shown for all combination of areas: SC, S1, M1, and PM. Significant $(p<0.01)$ covariance of SEPs during a given behavioral epoch is shown by open circles; filled circles indicate nonsignificant coefficient. $\Delta$ rrepresents the depth of modulation (difference between the maximal and minimal rvalue during task).C, Cue; D, instructed delay; $A c t M$, active movement; $H$, hold; Pass M, passive movement R, rest. C, Strength of covariance between the four recorded areas shown as no (dotted line), moderate (thin solid line), or strong (heavy line) correlation in each behavioral epoch. 
tory activity, such as SMA (Aizawa and Tanji, 1994). Both M1 and PM have many neurons exhibiting robust increased activities during instructed delay periods and this activity is positively correlated with the performance of upcoming movement (Wise, 1985; Alexander and Crutcher, 1990; Requin et al., 1990; Boussaoud, 1995). Therefore, the function of such a mechanism could be to protect the fidelity of such preparatory neural activity. Our monkeys were trained to move quickly when the go signal was presented to obtain reward. The stimuli applied to SR during the task were delivered at a fixed frequency of $3 \mathrm{~Hz}$ and provided no useful information for making successful trials. Such task-irrelevant activations of M1 and PM could result in the lower fidelity of the preparatory neural activity and disrupt the cortical mechanisms generating the motor-set for quick movement during the delay period. Therefore, suppressing such task-irrelevant activation would be beneficial for better performance of the subsequent movement. It is likely, therefore, that sensory gating before movement could attenuate irrelevant input to motor cortices and help motor cortex neurons achieve a better motor-set for the upcoming movements. This hypothesis is consistent with the observation that active extensions with faster reaction times were preceded by instructed delay periods with smaller SEPs.

Another mechanism that could be involved in modulating SEP amplitude is occlusion. If cells in a region are more active in a particular behavior, the responses evoked by extraneous input could be reduced because the evoked postsynaptic currents are subject to greater shunting. The degree to which this may be a contributing factor in gating remains for future resolution.

\section{Modulation of covariation of SEPs}

The observations on covariation of the SEPs are based on one recording site for each area and so are somewhat preliminary. Nevertheless, the results show that a sustained covariation of SEPs occurred in M1 and PM throughout the task, and transient covariation occurred during specific epochs for other sites, like S1 and SC. This demonstrates the power of documenting SEP correlations to further elucidate the neural mechanism underlying the modulation of sensory input during voluntary movement. It would be desirable to record from more sites per area simultaneously to characterize each region and the interactions among them more completely.

\section{Conclusion}

In summary, we found that responses to afferent inputs from cutaneous receptors are suppressed at several levels in a taskdependent manner. The strongest suppression occurred during dynamic limb movement and was due at least in part to top-down suppression (mediated by presynaptic and possibly postsynaptic inhibition), since it precedes the onset of active movement. This creates a general suppression because it was observed at the earliest relay in the spinal cord as well as all three cortical areas. After onset of active movement and during active hold, bottom-up mechanisms of lateral inhibition could also contribute to the observed reduction of SEPs. Preparation for an upcoming movement was associated with suppression of SEPs specifically in the motor cortical areas and the magnitude of this suppression was inversely correlated with the reaction time of the subsequent movement.

\section{References}

Aizawa H, Tanji J (1994) Corticocortical and thalamocortical responses of neurons in the monkey primary motor cortex and their relation to a trained motor task. J Neurophysiol 71:550-560.

Alexander GE, Crutcher MD (1990) Preparation for movement: neural representations of intended direction in three motor areas of the monkey. J Neurophysiol 64:133-150.

Andreatta RD, Barlow SM (2003) Movement-related modulation of vibrotactile detection thresholds in the human orofacial system. Exp Brain Res 149:75-82.

Angel RW, Malenka RC (1982) Velocity-dependent suppression of cutaneous sensitivity during movement. Exp Neurol 77:266-274.

Bays PM, Flanagan JR, Wolpert DM (2006) Attenuation of self-generated tactile sensations is predictive, not postdictive. PLoS Biol 4:e28.

Blakemore SJ, Wolpert DM, Frith CD (1998) Central cancellation of selfproduced tickle sensation. Nat Neurosci 1:635-640.

Boussaoud D (1995) Primate premotor cortex: modulation of preparatory neuronal activity by gaze angle. J Neurophysiol 73:886-890.

Chapman CE, Jiang W, Lamarre Y (1988) Modulation of lemniscal input during conditioned arm movements in the monkey. Exp Brain Res 72:316-334.

Cohen LG, Starr A (1987) Localization, timing and specificity of gating of somatosensory evoked potentials during active movement in man. Brain 110:451-467.

Coulter JD (1974) Sensory transmission through lemniscal pathway during voluntary movement in the cat. J Neurophysiol 37:831-845.

Curtis DR, Eccles JC (1960) Synaptic action during and after repetitive stimulation. J Physiol 150:374-398.

Eccles JC, Schmidt RF, Willis WD (1963) Depolarization of the central terminals of cutaneous afferent fibers. J Neurophysiol 26:646-661.

Ghez C, Pisa M (1972) Inhibition of afferent transmission in cuneate nucleus during voluntary movement in the cat. Brain Res 40:145-155.

Ghosh S, Porter R (1988) Corticocortical synaptic influences on morphologically identified pyramidal neurones in the motor cortex of the monkey. J Physiol 400:617-629.

Hantman AW, Jessell TM (2010) Clarke's column neurons as the focus of a corticospinal corollary circuit. Nat Neurosci 13:1233-1239.

Haugland M (1996) A flexible method for fabrication of nerve cuff electrodes. Paper presented at 18th Annual International Conference of the IEEE Engineering in Medicine and Biology Society, Amsterdam, November.

Hultborn H, Illert M, Nielsen J, Paul A, Ballegaard M, Wiese H (1996) On the mechanism of the post-activation depression of the H-reflex in human subjects. Exp Brain Res 108:450-462.

Jiang W, Lamarre Y, Chapman CE (1990) Modulation of cutaneous cortical evoked potentials during isometric and isotonic contractions in the monkey. Brain Res 536:69-78.

Lemon RN, van der Burg J (1979) Short-latency peripheral inputs to thalamic neurones projecting to the motor cortex in the monkey. Exp Brain Res 36:445-462.

Milne RJ, Aniss AM, Kay NE, Gandevia SC (1988) Reduction in perceived intensity of cutaneous stimuli during movement: a quantitative study. Exp Brain Res 70:569-576.

Mountcastle VB (1957) Modality and topographic properties of single neurons of cat's somatic sensory cortex. J Neurophysiol 20:408-434.

Murase N, Kaji R, Shimazu H, Katayama-Hirota M, Ikeda A, Kohara N, Kimura J, Shibasaki H, Rothwell JC (2000) Abnormal premovement gating of somatosensory input in writer's cramp. Brain 123:1813-1829.

Perlmutter SI, Maier MA, Fetz EE (1998) Activity of spinal interneurons and their effects on forearm muscles during voluntary wrist movements in the monkey. J Neurophysiol 80:2475-2494.

Requin J, Lecas JC, Vitton N (1990) A comparison of preparation-related neuronal activity changes in the prefrontal, premotor, primary motor and posterior parietal areas of the monkey cortex: preliminary results. Neurosci Lett 111:151-156.

Rushton DN, Rothwell JC, Craggs MD (1981) Gating of somatosensory evoked potentials during different kinds of movement in man. Brain 104:465-491.

Seki K, Fetz EE (2010) Modulation of sensory responses at spinal and cortical levels during preparation and execution of voluntary movement. Soc Neurosci Abstr 36:494.422.

Seki K, Perlmutter SI, Fetz EE (2003) Sensory input to primate spinal cord is 
presynaptically inhibited during voluntary movement. Nat Neurosci 6:1309-1316.

Seki K, Perlmutter SI, Fetz EE (2009) Task-dependent modulation of primary afferent depolarization in cervical spinal cord of monkeys performing an instructed delay task. J Neurophysiol 102:85-99.

Shergill SS, Samson G, Bays PM, Frith CD, Wolpert DM (2005) Evidence for sensory prediction deficits in schizophrenia. Am J Psychiatry 162: $2384-2386$.

Starr A, Cohen LG (1985) 'Gating' of somatosensory evoked potentials begins before the onset of voluntary movement in man. Brain Res 348: 183-186.

Thilo KV, Santoro L, Walsh V, Blakemore C (2004) The site of saccadic suppression. Nat Neurosci 7:13-14.
Tracey DJ, Asanuma C, Jones EG, Porter R (1980) Thalamic relay to motor cortex: afferent pathways from brain stem, cerebellum, and spinal cord in monkeys. J Neurophysiol 44:532-554.

Tsumoto T, Nakamura S, Iwama K (1975) Pyramidal tract control over cutaneous and kinesthetic sensory transmission in the cat thalamus. Exp Brain Res 22:281-294.

Urbain N, Deschênes M (2007) Motor cortex gates vibrissal responses in a thalamocortical projection pathway. Neuron 56:714-725.

Wasaka T, Nakata H, Kida T, Kakigi R (2005) Changes in the centrifugal gating effect on somatosensory evoked potentials depending on the level of contractile force. Exp Brain Res 166:118-125.

Wise SP (1985) The primate premotor cortex: past, present, and preparatory. Annu Rev Neurosci 8:1-19. 